2017-09-14

\title{
Modified inertial mass from information
}

\section{loss}

\section{McCulloch, ME}

http://hdl.handle.net/10026.1/10355

10.1142/S0217732317501486

Modern Physics Letters A

World Scientific Pub Co Pte Lt

All content in PEARL is protected by copyright law. Author manuscripts are made available in accordance with publisher policies. Please cite only the published version using the details provided on the item record or document. In the absence of an open licence (e.g. Creative Commons), permissions for further reuse of content should be sought from the publisher or author. 


\title{
Modified inertial mass from information loss.
}

\author{
Michael E. McCulloch* and Jaume Giné ${ }^{\dagger}$
}

November 30, 2017

\begin{abstract}
A modification of inertia (called MiHsC or quantised inertia) has been proposed that assumes that inertia is caused by Unruh radiation, and that this radiation is made inhomogeneous in space by either Rindler horizons caused by acceleration, or the distant Hubble horizon. The former predicts the standard inertial mass, and the later predicts galaxy rotation without dark matter and cosmic acceleration without dark energy. It is proposed here that this model can be derived in an alternative way by assuming that the sum of mass (M), energy (E) and the information content of horizons (I) is conserved (EMI) so that mass-energy is released in a discrete manner when the area of a Rindler horizon reduces. This model could be tested by looking for the quantisation of inertial mass and acceleration at very high accelerations, and may provide an explanation for the cosmological constant problem.
\end{abstract}

\section{Introduction}

Arguably, the most significant problems in physics today are the galaxy rotation problem (discovered by [25] and [19]) and cosmic acceleration (discovered by [18] and [17]). These have been modelled in the past by the arbitrary addition of dark matter and dark energy, but the arbitrariness of this approach is unappealing. A less arbitrary solution is MoND (Modified Newtonian Dynamics) ([15]) that empirically suggested a minor change to the laws of either gravity or inertia, but MoND is also partly arbitrary since an adjustable paramtere is needed.

McCulloch [11, 14] proposed a new non-arbitrary model for inertia (called quantised inertia or MiHsC: Modified inertia due to a Hubble scale Casimir effect) that assumes that the inertia of an object is due to the Unruh radiation [24] it sees when it accelerates. The Rindler horizon that appears in the opposite direction to its acceleration damps the Unruh radiation on that side of the object producing a radiation pressure that looks like inertial mass [14]. Also, when accelerations are extremely low the Unruh waves become very long and are also

\footnotetext{
*Plymouth University, Plymouth, PL4 8AA, mike.mcculloch@plymouth.ac.uk

†Universitat de Lleida, Catalonia, Spain, gine@matematica.udl.cat
} 
damped, this time in all directions, by the Hubble horizon (Hubble-scale Casimir effect). This leads to a new loss of inertia as accelerations become tiny. MiHsC modifies the standard inertial mass $(m)$ to a modified one $\left(m_{i}\right)$ as follows:

$$
m_{i}=m\left(1-\frac{2 c^{2}}{|a| \Theta}\right)
$$

where $\mathrm{c}$ is the speed of light, $\Theta$ is twice the Hubble distance, ' $|\mathrm{a}|$ ' is the magnitude of the relative acceleration of the object relative to surrounding matter. Eq. 1 predicts that for terrestrial accelerations $\left(\mathrm{eg}: 9.8 \mathrm{~m} / \mathrm{s}^{2}\right.$ ) the second term in the bracket is tiny and standard inertia is recovered, but in low acceleration environments, for example at the edges of galaxies (when a is tiny) the second term in the bracket becomes larger and the inertial mass decreases in a new way so that $\mathrm{MiHsC}$ can explain galaxy rotation without dark matter [13] and cosmic acceleration without dark energy [11, 12].

The concept of holographic physics was developed by t'Hooft [22]. It has been suggested in $[7,8]$ that $\mathrm{MiHsC}$ and the holographic approaches have a link, and in this paper a possible link is proposed. In the next section we present the main result of this paper which is an alternative derivation of Eq. 1 using Landauer's principle, which quantifies the production of heat from information loss.

\section{Method \& Results}

Normally it is assumed that mass-energy is conserved, but it is proposed here that what is actually conserved is the sum of energy-mass $\left(E=m c^{2}\right)$ and information, something that can be referred to as EMI. The concept of information is fairly new in physics. Maxwell described the thought experiment in which molecules in a box with a partition dividing it can be sorted by a 'demon' who controls a door in the partition into fast moving molecules on the right of the partition and slow moving ones on the left. To do this the demon needs information about the molecules' speed and position. This appeared to show that information can be used to decrease the entropy in the box, in violation of the second law of thermodynamics.

The paradox was resolved by Landauer [10] who was studying computer memory and realised that a computer's memory is a physical system, with the bits corresponding to physical attributes, and so when memory is erased a complex pattern of information (the former state, eg: 010011) is suddenly made uniform (000000) and since this is also a physical system, there is a very real reduction of entropy which must be associated with a release of high-entropy heat to satisfy the second law of thermodynamics. Therefore Maxwell's demon releases heat whenever he clears his memory of one molecule's data to look at another, and by doing that he must increase the heat and entropy of the cosmos more than he decreases the entropy within the box.

This is now called Landauer's principle and it specifically states that if an observer looses information about a physical system then they must lose the abil- 
ity to extract some work from that system, the work turns to unusable heat. The energy lost (converted to heat) by the deletion of a bit of information is $E=k T \ln 2$ where $\mathrm{k}$ is Boltzmann's constant and $\mathrm{T}$ is the ambient temperature. This principle has now been confirmed experimentally [2, 9].

The energy lost from the deletion of $\mathrm{N}$ bits of information is $E=k T N \ln 2$ and so assuming the conservation of EMI we can write down the initial and final EMI content, for a system that changes its information content from $N_{1}$ to $N_{2}$ as follows

$$
m_{1} c^{2}+k T N_{1} \ln 2=m_{2} c^{2}+k T N_{2} \ln 2
$$

We can rearrange this as follows

$$
m_{2}-m_{1}=d m=\frac{k T}{c^{2}}\left(N_{1}-N_{2}\right) \ln 2=\frac{k T}{c^{2}} N_{1}\left(1-\frac{N_{2}}{N_{1}}\right) \ln 2
$$

Using the concept of holographic physics we can assume that the amount of information is related to the number of Planck areas that are on the closest event horizon seen by the object. In the direction of acceleration the information horizon is the Hubble horizon and its area will not change, it will remain half the Hubble sphere. In contrast the horizon in the opposite direction to the acceleration will first be half the area of the Hubble horizon because the object is not yet accelerating. The information content is then the number of Planck areas we can fit into half the Hubble horizon, which is its surface area over the Planck area

$$
N_{1}=\frac{2 \pi R_{U}^{2}}{l_{P}^{2}}
$$

where $l_{P}$ is the Planck length and $R_{U}$ is the radius of the Hubble sphere. If the object then accelerates, this horizon then shrinks to become a Rindleracceleration horizon instead. Less information (fewer Planck areas) can be encoded on that Rindler horizon and therefore a certain amount of energy must be released as heat following Landauer's principle. If we assume for simplicity that the Rindler horizon is a hemisphere of radius $d$, then the information becomes

$$
N_{2}=\frac{2 \pi d^{2}}{l_{P}^{2}}
$$

Substituting these into Eq. 3 we get

$$
d m=\frac{k T}{c^{2}} N_{1}\left(1-\frac{d^{2}}{R_{U}^{2}}\right) \ln 2
$$

The distance to the Rindler horizon is $d=c^{2} / a$ and $R_{U}=\Theta / 2$ so we have 


$$
d m=\frac{k T}{c^{2}} N_{1}\left(1-\frac{4 c^{4}}{a^{2} \Theta^{2}}\right) \ln 2
$$

Now we consider the case when $1-4 c^{4} /\left(a^{2} \Theta^{2}\right)$ is small so that $a \sim 2 c^{2} / \Theta$, in other words we assume cosmologically-low accelerations. Now we use the approximation $\rho \sim-\ln (1-\rho)$ when $\rho$ is sufficiency small and we get

$$
d m=-\frac{k T}{c^{2}} N_{1} \ln \left(\frac{4 c^{4}}{a^{2} \Theta^{2}}\right) \ln 2
$$

Now since the movement of the accelerating particle is defined by a threedimensional space with a three-vector velocity in order to compute the average kinetic energy we have three degree of freedom and hence we have that the total energy by the equipartition rule is

$$
E=\frac{3}{2} N_{1} k T=m c^{2}
$$

and substituting into Eq. 8 we obtain

$$
d m=-\frac{4}{3} m \ln \left(\frac{2 c^{2}}{a \Theta}\right) \ln 2
$$

If we add and subtract 1 inside the bracket

$$
d m=-\frac{4}{3} m \ln \left(1+\frac{2 c^{2}}{a \Theta}-1\right) \ln 2
$$

Since $\ln (1+x) \sim x$ for small $\mathrm{x}$ we can write

$$
d m=-\frac{4}{3} m\left(\frac{2 c^{2}}{a \Theta}-1\right) \ln 2
$$

Which leaves

$$
d m=\frac{4}{3} m\left(1-\frac{2 c^{2}}{a \Theta}\right) \ln 2
$$

Taking into account that $\frac{4}{3} \ln 2 \sim 0.92$, this is the amount of energy or mass released when a body accelerates from zero (or at least the minimum acceleration predicted by quantised inertia) to a larger acceleration 'a'. It is similar to the expression for quantised inertia $(\mathrm{MiHsC})$, which is $\mathrm{Eq}$. 1, but is only valid when $2 c^{2} / a \Theta-1=$ small so that $a \sim 2 c^{2} / \Theta$ in other words for cosmologicallylow accelerations. One definite source of error in this derivation, and which may account for the different numerical factors in Eq. 13 when compared to Eq. 1, is the assumption that the Rindler horizon is a sphere centred on the accelerating body. The Rindler horizon is a more complex shape and this needs a more accurate mathematical model. 


\section{Discussion}

Since inertial mass comes from the loss of Planck areas, then the Rindler horizon can never be smaller than the Planck area and this predicts a maximum possible acceleration. A similar maximum acceleration was derived by Sakharov [23] using just Unruh radiation and assuming a maximum possible temperature [5]. MiHsC already predicts there is a minimum acceleration of $2 c^{2} / \Theta \sim 7 \times 10^{-10} \mathrm{~m} / \mathrm{s}^{2}$ since at that low acceleration the Rindler horizon expands to the Hubble horizon and can't expand further. This prediction agrees with the magnitude of the recently observed cosmic acceleration [12].

The new prediction here is that the area of the Rindler horizon cannot be less than a Planck area, since that area is the smallest area that is observable, so that we have for the smallest horizon, radius $\mathrm{d}$

$$
2 \pi d^{2} \sim l_{P}^{2}
$$

where $d=c^{2} / a_{\max }$ where $a_{\max }$ is the maximum acceleration. Therefore

$$
a_{\max } \sim \frac{\sqrt{2 \pi} c^{2}}{l_{P}} \sim 1.4 \times 10^{52} \mathrm{~m} / \mathrm{s}^{2}
$$

Theoretically such a maximum acceleration would also fix the problem of infinite divergences in black holes pointed out by 't Hooft [21] and Susskind and Uglum [23]. This maximum acceleration is close to that derived in a very different way, from quantum mechanics, by Caianiello et al. [3,4] and references therein.

\section{A Test}

This model might be tested by looking for a quantisation of inertial mass, and accelerations, at very high acceleration. According to the derivation above, at high accelerations the Rindler horizon seen by the accelerated object is made up of an integer number of Planck areas and therefore it can only shrink in discrete areal jumps. This means that only accelerations corresponding to a whole number of Planck areas on the horizon are allowed and the accelerated object should jump between these accelerations. The proportionate (dimensionless) size of the jump $(\mathrm{J})$ relative to the acceleration itself is given by the size of a Planck area divided by the area of the horizon (as used above), as follows.

$$
J=\frac{l_{P}^{2}}{2 \pi d^{2}}=\frac{l_{P}^{2} a^{2}}{2 \pi c^{4}}=5 \times 10^{-105} a^{2}
$$

For a typical terrestrial acceleration $\mathrm{J}$ is of the order of $10^{-103}$ and for an acceleration of $a_{\max }$ then $\mathrm{J}$, of course, approaches 1 . The high accelerations required to make $\mathbf{J}$ significant enough to test this, close to $a_{\max }$ are difficult to 
achieve, although Papini et al. [16] suggested that light resonating in a cavity and type I superconductors may have accelerations this large.

Further, if we replace the size of the Rindler horizon in Eq. 16 with the diameter of the cosmic horizon, and therefore look at the relative size of the acceleration jumps required due to the discreteness of the cosmic horizon as the universe expands, then we get

$$
J=\frac{l_{P}^{2}}{4 \pi \Theta^{2}}
$$

Using the co-moving cosmic diameter: $\Theta=8.8 \times 10^{26} \mathrm{~m}$, Eq. 17 predicts that $J=2.63 \times 10^{-125}$ which is similar to the observed ratio between the cosmological energy density and the Planck energy density which is $10^{-122}$ (see eg: [6]) (the cosmological constant problem).

\section{Conclusions}

A new theory of inertial mass called quantised inertia or MiHsC predicts wellknown anomalies in astrophysics such as the galaxy rotation problem and cosmic acceleration. It is shown here to be derivable, within $8 \%$, by assuming the conservation of a sum of energy, mass and holographic information. In this scheme, information, stored as Planck areas on Rindler horizons, is lost as objects accelerate and form smaller Rindler horizons. The lost information then appears via Landauer's principle as mass-energy.

This proposal may provide an explanation for the cosmological constant problem, and could be tested by looking for a quantisation of inertia and accelerations at very high accelerations.

\section{Acknowledgements}

The second author is partially supported by a MINECO/ FEDER grant number MTM2014-53703-P and an AGAUR (Generalitat de Catalunya) grant number 2014SGR 1204.

\section{Note}

This is the authors' accepted manuscript. The final published version of this work (the version of record) is published by World Scientific in Modern Physics Letters A available at: https://doi.org/10.1142/S0217732317501486. This work is made available online in accordance with the publisher's policies. Please refer to any applicable terms of use of the publisher. 


\section{References}

[1] Bennett, C.H., Demons, Engines and the Second Law, Scientific American 257 (1987), no. 5, 108-116.

[2] Berut, A., A. Arakelyan, A. Petrosyan, S. Ciliberto, R. Dillenschneider, E. Lutz, Experimental verification of Landauer's principle linking information and thermodynamics, Nature 483 (2012), 187-190.

[3] Caianiello E.R., Is There A Maximal Acceleration? Lett. Nuovo Cim. 32 (1981) 65-75.

[4] Caianiello, E.R., Feoli, A., Scarpetta, G., Capozziello, S., De Ritis, R. Maximal acceleration tunneling from 'nothing', Int. J. Mod. Phys. D 3 (1994) 485-492.

[5] Feoli, A., Papini, G., Scarpetta, G., Maximal acceleration effect or photons in cavity resonators, in Recent developments in General Relativity, 2000, pp. $405-409$.

[6] Funkhouser, S., 2006. A new large-number coincidence and a scaling law for the cosmological constant. Proc. of the Royal Society A., Vol. 464, 2093.

[7] Giné, J., The holographic scenario, the modified inertia and the dynamics of the universe, Modern Physics Letters A 27 (2012), no. 34, 1250208.

[8] Giné, J., Cosmological consequences of the holographic scenario, Int. J. Theor. Phys., 52 (2013), no. 1, 53-61.

[9] Jun, Y., Gavrilov, M. Bechhoefer, J., High-precision test of Landauer's principle in a feedback trap, Phys. Rev. Lett. 113 (2014), no. 19, 190601.

[10] Landauer, R., Irreversibilty and heat generation in the computing process, IBM Journal of Research and Development. 5 (1961), no. 3, 183-191.

[11] McCulloch, M.E., The Pioneer anomaly as modified inertia, MNRAS 376 (2007), 338-342.

[12] McCulloch, M.E., Minimum accelerations from quantised inertia, EPL 90 (2010), 29001.

[13] McCulloch, M.E., Testing quantised inertia on galactic scales, Astr. \& Space Sci. 342 (2012), 575-578.

[14] McCulloch, M.E., Inertia from an asymmetric Casimir effect, EPL 101 (2013), 59001.

[15] Milgrom, M., 1983. A modification of the Newtonian dynamics as a possible alternative to the hidden mass hypothesis. Astrophys. J., 270, 365. 
[16] Papini G., Feoli, A., Scarpetta, G., Maximal acceleration of photons with effective mass. Frequency shift in cavity resonators, Phys. Lett. A 220 (1995), 50-53.

[17] Perlmutter, S., et al., 1999. Astrophys. J., 517, 565.

[18] Riess A.G., 1998. Astron. J., 116, 1009.

[19] Rubin, V., N. Thonnard and W.K. Ford Jr., 1980. Rotational properties of $21 \mathrm{SC}$ galaxies with a large angle of luminosities and radii from NGC4605 $(\mathrm{R}=4 \mathrm{kpc})$ to $\mathrm{UGC} 2885(\mathrm{R}=122 \mathrm{kpc})$. Astrophys.J., 238,471 .

[20] Sakharov, A.D., Maximum termperature of thermal radiation, JETP Lett. 3 (1966), 288.

[21] 't Hooft, G., On the quantum structure of a black hole, Nucl. Phys. B 256 (1985) $256,727-745$.

[22] 't Hooft, G., Dimensional reduction in quantum gravity. arXiv:grqc/9310026, preprint, 1993.

[23] Susskind, L., Uglum. J., Black Hole Entropy in Canonical Quantum Gravity and Superstring Theory, Phys. Rev. D 50 (1994) 2700-2711.

[24] Unruh, W.G., Notes on black-hole evaporation Phys. Rev. D 14 (1976), 870.

[25] Zwicky, F., DerRotverschiebung von extragalaktischen Nebeln. Phys. Acta., 6,110 . 